\title{
Clinical Features and Histopathological Spectrum in Adolescent Onset Nephrotic Syndrome in a Romanian Children Population
}

\author{
Gall Zsuzsanna1 ${ }^{1}$, Duicu Carmen², Kiss Eva², Egyed-Zsigmond I³, Cucerea Manuela ${ }^{1}$, Simon Marta ${ }^{1}$ \\ 1 Department of Pediatrics IV, University of Medicine and Pharmacy, Târgu-Mureș \\ 2 Department of Pediatrics II, University of Medicine and Pharmacy, Târgu-Mures \\ ${ }^{3}$ Department of Pathology, University of Medicine and Pharmacy, Târgu-Mureș
}

\begin{abstract}
Objectives of study: The adolescent population signifies the transitory period where the frequent occurrence of different histopathological lesions in patients with nephrotic syndrome (NS) is different from that seen in young children as well as that seen in adults. This study aimed to analyze the clinical features and histopathological spectrum of adolescent-onset NS. Material and methods: We retrospectively evaluated clinical features, biochemical investigations and histopathology of 103 children with idiopathic NS referred to the Pediatric Department, County Hospital of Târgu-Mureș. Fourteen patients with congenital-, infantil- and secondary NS were excluded from this study. Results: The patients were divided into 2 groups: in group A we included 69 patients diagnosed with NS diagnosed before 10 years-old, with a median age of $3.76 \pm 1.96$ years, majority males (59.42\%) and presenting the pure form of NS. On the other hand, in group B we included 20 adolescents having the median age at the onset of the disease $13.61 \pm 2.18$ years, with equal distribution of the sexes and presenting the impure form in $65 \%$ of cases. The majority of the patients in both groups $(68.11 \%$ and $70 \%$ respectively) responded to steroid therapy. The commonest histopathological subtype in both groups was focal segmental glomerulosclerosis. Conclusions: The incidence of nephrotic syndrome has increased in the last years. The impure form of NS is more frequent in the adolescents than in younger patients. Adolescents with impure and steroid-resistant NS at presentation have other lesions than minimal change disease. The early genetic diagnosis in NS is important for proper clinical management of the patients, prognosis and genetic counseling of the families.
\end{abstract}

Key words: nephrotic syndrome, adolescent, biopsy

Received: 24 April 2014 / Accepted 12 August 2014

\section{Introduction}

Nephrotic syndrome (NS) describes the collection of clinical and laboratory findings secondary to glomerular dysfunction and resulting in severe proteinuria. NS affects about 2-7/100000 children annually, the cumulative prevalence rate being approximately 16 cases $/ 100000$ people in USA $[1,2,3]$, while the incidence of NS in Romania is not known.

In the literature, there are very few studies addressing the problem of nephrotic syndrome in adolescents. The adolescent population signifies the transitory period where the frequent occurrence of different histopathological lesions in patients with nephrotic syndrome is different from that seen in young children as well as that seen in adults $[4,5]$. Approximately $85 \%$ of children present primary NS, associated with "minimal change disease" (MCD) in 90\%

Correspondence to: Zsuzsanna Gáll

E-mail: nzsuzsanna@yahoo.com of cases and being associated usually with a good prognosis, $93 \%$ of cases entering complete remission after 4-8 weeks of corticosteroid theapy $[3,6,7]$. Other histological types like mesangioproliferativ glomerulonephritis and focal-segmental glomerulosclerosis (FSGS) is more frequent in adolescents [4]. It may present as primary NS, however the prognosis associated with this form is poor, only $30 \%$ of cases achieving a complete remission and often being complicated by steroid-resistance [2]. On the other hand, adult-onset NS has been associated with a higher prevalence of hypertension, renal impairment and a slower response to steroids, but a lower tendency to relapse $[2,8,9]$. Hypertension in NS appears more frequent in teenagers than in younger children $[2,10]$.

\section{Objectives of study}

The aim of this retrospective study was to analyze the clinical features and histopathological spectrum of adolescentonset NS in a Romanian population. 


\section{Material and methods}

We reviewed the medical records of 103 children with nephrotic syndrome fulfilling the International Study of Kidney Diseases in Childhood (ISKDC) criteria referred to the Pediatric Department, County Hospital of TârguMureş, between January 2001 and December 2012. Pertinent data, including age at the onset of the disease, gender, clinical features, biochemical investigations, histopathology, frequency of the relapses, type of prescribed medications and the disease outcome for a minimum of 1 year follow-up were obtained in all the patients.

Inclusion criteria in the study were: diagnosis of NS, defined as proteinuria $\left(>40 \mathrm{mg} / \mathrm{m}^{2} / \mathrm{h}\right)$ and hypoalbuminaemia $(<25 \mathrm{~g} / \mathrm{l})$, edema and hyperlipidemia, having its onset between 1 to 18 years of age.

Exclusion criteria were the onset of the disease in the first year of life (congenital and infantile NS) or in adulthood. Congenital and infantile NS has different etiology and histopathologic characteristics, therefore 14 patients with this diagnosis were excluded from this study. Patients with clinical or laboratory features incompatible with primary NS, such as secondary nephrotic syndrome were excluded from this study.

On each visit, the children were evaluated clinically for evidence of disease activity and complications (infections and drug side-effects). Hypertension was defined as systolic or diastolic blood pressure $>95$ th percentile for age, gender and height on three separate occasions.

The patients were classified in two study groups: group A: 69 patients with NS diagnosed before 10-years-old (reference group), and group B - 20 adolescent patients.

All the investigations were in accordance with the Declaration of Helsinki.

\section{Statistical analysis}

Statistical analysis was performed with Microsoft Excel 2007 and Graph Pad In-stat software version 3.0. Data were labeled as nominal or quantitative variables. Nomi- nal variables were characterized by means of frequencies. Quantitative variables were tested for normality of distribution using Kolmogorov-Smirnov test and were described by mean \pm standard deviation or median and range (minimum, maximum), whenever appropriate. The frequencies of nominal variables were compared with a chi-square test and Fisher's exact test. Differences in the mean or median between groups were analyzed using the Student t-test, Mann-Whitney U-test. The level of statistical significance was set at $\mathrm{p}<0.05$.

\section{Results}

In the last years we observed a significant increase in the number of new cases of nephrotic syndrome (NS): 35 patients $(39.32 \%)$ presented the onset of the disease after 2008 (Figure 1). The incidence of NS in adolescent from all the NS diagnosed at all ages was $19.42 \%$.

The demographic, clinical and paraclinical data at the onset of the disease are described in Table I. In A group the median age at the diagnosis of the nephrotic syndrome was $3.76 \pm 1.96$ years (range: 12.5 months -8.2 years); with the predominance of males $(56.52 \%)$. On the other hand, in B group the median age at the onset of the disease was $13.61 \pm 2.18$ years (range: $10.06-17.05$ years), with equal distribution of the sexes.

The majority of patients in both groups $(68.11 \%$ and $70 \%$, respectively) was diagnosed with steroid-sensitive nephrotic syndrome. In the adolescent group 6 patients (30\%) had steroid-resistant nephrotic syndrome, 3 of them presenting the onset of the massive proteinuria after 2008 (Figure 1).

In group A the majority of the patients (59.42\%) presented the pure form of NS (without hypertension, haematuria or BUN retention). On the other hand, in the adolescent group only 7 patients presented pure NS, 13 patients $(65.00 \%)$ had impure nephrotic syndrome, all of them presenting hypertension and 5 patients $(27.77 \%)$ microscopic haematuria.

Table I. Demographic characteristics, clinical and laboratory data at the time of diagnosis in group A (1-10 years) and group B (>10 years).

\begin{tabular}{|c|c|c|c|}
\hline Clinical caracteristics & Group A & Group B & $\mathrm{p}$ value \\
\hline Number of cases & 69 & 20 & - \\
\hline Age (year) mean $\pm \mathrm{SD}$ & $3.76 \pm 1.96$ & $13.61 \pm 2.18$ & $<0.0001$ \\
\hline Sex ratio (Male:Female) no & $39: 30$ & $10: 10$ & $>0.05$ \\
\hline SSNS:SDNS:SRNS no & $47: 3: 19$ & 14:0:6 & $>0.05$ \\
\hline Pur:impur no & $41: 28$ & $7: 13$ & $>0.05$ \\
\hline Proteinemia $(g / l)$ mean $\pm S D$ & $45.0 \pm 10.3$ & $47.3 \pm 10.3$ & $>0.05$ \\
\hline Proteinuria (mg/m²/h) median (range) & $\begin{array}{c}155.3 \\
(43.8-1918)\end{array}$ & $\begin{array}{c}155.1 \\
(65.5-338.2)\end{array}$ & $>0.05$ \\
\hline Serum cholesterol $(\mathrm{mmol} / \mathrm{l})$ mean $\pm \mathrm{SD}$ & $10.5 \pm 3.98$ & $10.8 \pm 3.40$ & $>0.05$ \\
\hline Serum triglycerid (mmol/l) median (range) & $2.4(0.46-8.56)$ & $2.8(0.44-8.89)$ & $>0.05$ \\
\hline Serum creatinine (mg/dl) median (range) & $0.48(0.2-8.3)$ & $0.8(0.4-2.23)$ & $<0.0001$ \\
\hline Creatinine clearance $\left(\mathrm{ml} / \mathrm{min} / 1.73 \mathrm{~m}^{2}\right)$ mean $\pm \mathrm{SD}$ & $1.4 \pm 0.55$ & $1.3 \pm 0.44$ & $>0.05$ \\
\hline Hypertonia (N:preHT:HTstl:HTstII) no & $45: 10: 9: 5$ & $7: 1: 8: 4$ & 0.007 \\
\hline Hematuria no (\%) & $18(26.08 \%)$ & $5(25.00 \%)$ & $>0.05$ \\
\hline Hystology (MCD:FSGS:MPGN:MGN) no & $1: 4: 2: 0$ & $0: 3: 2: 1$ & $>0.05$ \\
\hline
\end{tabular}




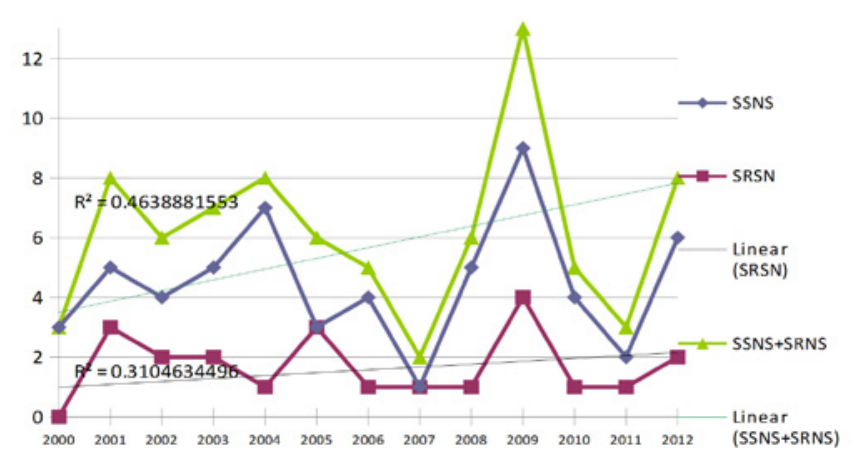

Figure 1. Correlations between nephrotic syndrome type and number of new cases of nephrotic syndrome/year. (SSNS: steroidsensitive nephrotic syndrome, SDNS: steroid-dependent nephrotic syndrome, SRNS: steroid-resistant nephrotic syndrome)

Clinical characteristics of the hypertension are presented in Table II. In the majority of the younger patients (75\%) hypertension was transient, on the other hand in the majority of the adolescents (53.85\%) this was persistent. Hypertension was found more frequently in the older patients $(65.00 \%$ in group $\mathrm{B}, 34.78 \%$ in group $\mathrm{A})$ $(p=0.007)$ and appeared more frequent in males $(54.00 \%)$ than in females, though the difference was not statistically significant $(p>0.05)$. In the study groups we found the following stages of hypertension: prehypertension in 11 cases $(29.73 \%)$ (median blood pressure $>90^{\text {th }}$ percentile, however $<95^{\text {th }}$ percentile for age, sex and height), stage I. hypertension in 17 cases $(45.92 \%)$ (median BP between $95-99^{\text {th }}$ percentiles for age, sex and height) (45.95\%), stage II. hypertension in 9 cases $(24.32 \%)$ (median BP $>99^{\text {th }}$ percentiles for age, sex and height): 9 cases and no case of hypertensive emergency. Blood pressure was monitored for 24 hours (ABPM) in 32 patients (86.48\%) of the 37 children with NS and hypertension, which confirmed elevated BP above the $90^{\text {th }}$ percentile. In $91.89 \%$ of children the diurnal index was lower than $8 \%$, while in $8.11 \%$ of the cases monitored by ABPM diurnal index was higher than $15 \%$. In these patients we noted the presence of overweight $\left(\mathrm{BMI}>25 \mathrm{~kg} / \mathrm{m}^{2}\right)$, which is an important associated risk factor in the development of hypertension. During the follow up only one patient developed a hypertensive complication, represented by mild hy-

Table II. Demographic and clinical caracteristics of the hypertension in patients with nephrotic syndrome.

\begin{tabular}{ccccc}
\hline $\begin{array}{c}\text { Hyperten- } \\
\text { sion }\end{array}$ & Group A (<10 years) & \multicolumn{2}{c}{ Group B (>10 years) } \\
\hline Male & Nr. & $\%$ & Nr. & $\%$ \\
Female & 13 & 54.17 & 7 & 53.85 \\
Persistent & 6 & 25.00 & 7 & 46.15 \\
Transient & 18 & 75.00 & 6 & 53.85 \\
PreHT & 10 & 41.67 & 1 & 46.15 \\
HT st I & 9 & 37.50 & 8 & 7.69 \\
HT st II & 5 & 20.83 & 4 & 61.54 \\
Total & 24 & 34.78 & 13 & 30.77 \\
\hline preHT - prehypertension - median blood pressure $>90$ th percentile, but < 95 th percentile for
\end{tabular}

(1) percentile for age, sex and height, HT st I - hypertension stage I - median BP between 95-99th percentiles, HT st II - hypertension stage I - median BP>99th percentiles pertensive retinopathy. No other organ damage was found in the rest of the cases (no hypertensive encephalopathy, hypertensive retinopathy or left ventricular hypertrophy).

Percutaneous renal biopsy was performed in 13 cases (14.61\% in the study groups; $10.69 \%$ in group A and $30.00 \%$ in group B). The biopsy specimens were studied by light- and electron-microscopy and immunofluorescency. The mean number of glomeruli studied was 13 (range 5-23). In both groups focal segmental glomerulosclerosis (FSGS) was the commonest histopathological subtype, occurring in $4(5.79 \%)$ and 3 patients $(15.00 \%)$ respectively (Figure 2). Other lesions included mesangioproliferative glomerulonephritis (MPGN) in 2 patients from each groups, membranous glomerulopathy (MGN) and minimal change disease (MCD) in one patient each. (Table I.)

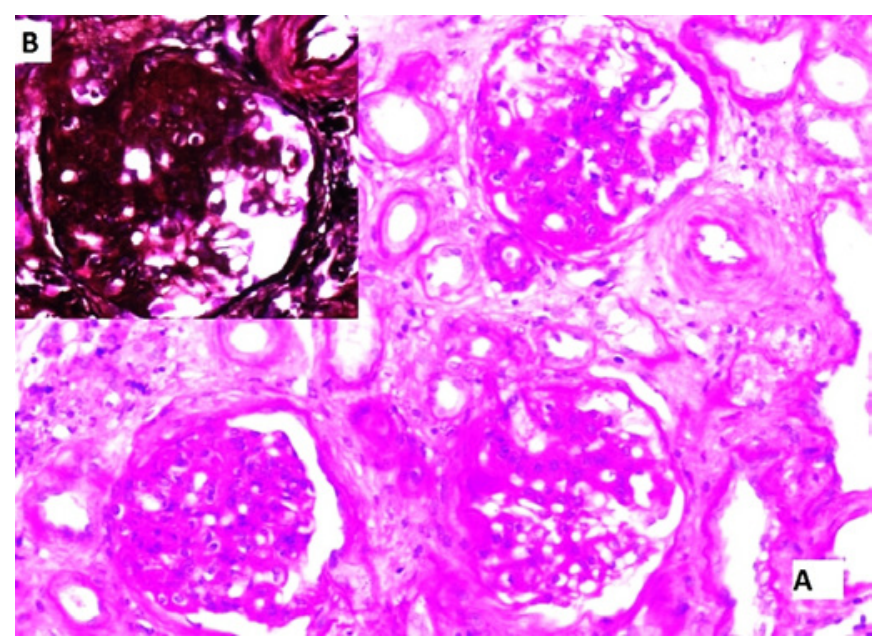

Figure 2. Light microscopic images of renal biopsy specimen. A - one glomerulus with global sclerosis (at 7 o'clock) and two gomeruli with perihilar segmental sclerosis (at 1 and 4 o'clock) (PAS stain, $x 400), B$ - a glomerulus with perihilar segmental sclerosis (Jones-stain, $x 400$ )

Three patients were lost to follow up due to low compliance. After a mean follow up of 92.48 months (1-12 years), $5.61 \%$ continued to have severe proteinuria, while $68.54 \%$ of the subjects achieved complete remission, though $11.29 \%$ presented frecvent relapses ( 2 or more relapses in the first 6 month, or 3 or more relapses in one year). In 7 patients (7.86\% of the patients with NS, $28 \%$ of the patients with SRNS) the disease progressed towards end-stage renal disease (ESRD). Three of these patients beneficiated from Continuous Ambulatory Peritoneal Dialysis (CAPD) and 4 patients from hemodialysis, while one patient received renal-transplant. One patient with SRNS who underwent initially CAPD followed by hemodialysis was lost. While in the younger children ESRD appeared only in $5.79 \%$, in the adolescents the disease progressed more frecvently towards renal insufficiency (15\%), though the difference was not significant $(p=0.18)$.

\section{Discussion}

There are few data regarding adolescent-onset NS [4]. This study was conducted to analyze for the first time the clinical 
spectrum and histopathological lesions of adolescent-onset NS in a romanian population, using the longest follow-up period published in the literature so far. The incidence of adolescent-onset NS in our study group was similar to the data published previously $[11,12]$.

While in younger children the ratio of males to females varies from 2:1 to 3:2 in different studies, in adolescents and adults the male-to-female prevalence is approximately equal as also confirmed in our study [2,3]. ISKDC data indicate that $66 \%$ of patients with either MCD or FSGS are male, whereas $65 \%$ of individuals with MPGN are female, confirmed also in our younger study group. In contrast, in our adolescent group all 3 patients with FSGS were female, and one of two patients with MPGN was male $[2,3,13]$.

According to the ISKDC report regarding the histopathological distribution of NS, $84.5 \%$ of children have MCD, 9.5\% had focal segmental glomerulosclerosis, $2.5 \%$ have mesangioproliferative glomerulonephrities and 3.5\% have membranous nephropathy or other cause of NS [3,6]. Similar distribution was observed in our younger group: FSGS $5.79 \%$ and MPGN $2.89 \%$. On the other hand, the histopathological distribution of NS in adults is very different from the one in childhood; there is a higher incidence of membranous nephropathy up to $50 \%$, whereas MCD only constitutes $25 \%$ of the total forms, with FSGS $15 \%[2,13]$. In our adolescent group $15 \%$ had FSGS, $10 \%$ MPGN and 5\% membranous glomerulopathy.

More studies have suggested a change in the histology of NS in the last decades, while its incidence has remained stable $[1,2,13]$. In contrast, other studies showed an increase in the number of children with new-onset NS and a significant increase in primary steroid resistance in the latter decade [14]. In the present study we observed a higher incidence of both SSNS and SRNS in both groups. The frequency of FSGS associated with NS seems to be increasing. A review of the literature suggested a doubling of the incidence of FSGS in the recent years [13], also confirmed in our study, as all of our adolescent patients with FSGS presented the onset of the disease after 2008.

Another classification in "pure" and "impure nephrotic syndromes", based on the clinical and biological features and the response to therapy and prognosis is useful for a clinician [15]. Impure NS is characterized by non-selective proteinuria, macroscopic and/or microscopic haematuria, frequent and persistent hypertension, nitrogen retention syndrome persisting after resuming the diuresis, low serum complement level and steroid resistance. In this study the incidence of impure NS was higher (46.07\%) compared to the $20 \%$ described in the literature [15] in both groups, and $65 \%$ in the older group. NS with later onset tends to manifest as an impure form. None of our patients with impure NS had low serum complement level. Arterial hypertension was a frequent finding in our study, especially in the adolescent group. Hypertension was persistent, though well-controlled, in $39.46 \%$ of our patients, observation also found by Ponticelli et al $[16,17]$.
The majority of the patients with hypertension had a diurnal index lower than $10 \%$, which is suggestive for secondary hypertension [17].

Corticosteroids have been used to treat idiopathic nephrotic syndrome [3], but, even with new corticosteroid regimens, $80-90 \%$ of children with SSNS have relapses, and nearly $50 \%$ relapse frequently $[2,6,18,19]$. In our study groups 16 patients $(17.98 \%)$ had no relapse and 43 children (48.32\%) presented two or more relapses, $11.29 \%$ presenting frequent relapses. Those children who receive repeated courses of steroid are at risk of the adverse effects of steroids.

SRNS with FSGS in children progress towards ESRD within 5 years from onset in $15-34 \%$ of cases [2,20,21]. In the present study two patients $(28.57 \%)$ out of seven with FSGS and SRNS developed ESRD, one of them early, at 13-years-old, 9 months after the onset of the illness. In this case genetic analysis confirmed a de novo WT1 gene mutation. After kidney transplantation the evolution of the patient was favorable, with diuresis according to intake, normalization of the blood pressure and the renal function tests and normal protein serum level. In this moment, 18 month after transplantation, the patient is in complete remission.

In SRNS cases it is recommended to effectuate histopathological examination of renal biopsy specimen and molecular genetic analysis too. Cyclosporin therapy is indicated in these cases, both for immunomodulating effect as well as for intrinsic role of reducing proteinuria. If within 3 months of treatment this proves to be ineffective or if the genetic substrate is confirmed, it is mandatory to suspend the cyclosporin therapy [6].

There were a number of limitations to our study, for example: there were deviations from the study protocol caused by patient and parent non-compliance (omitting medication, non-acceptance of the kidney biopsy, parents initially refused dialysis). Molecular genetic testing was only possible in 5 patients and renal biopsy in 13 children. A significant limitation to our study was its retrospective nature and the small sample size, however our findings might trigger future randomized trials on this topic.

\section{Conclusions}

According to literature data the incidence of nephrotic syndrome and steroid-resistant NS has increased in the second half of the last decade, observation confirmed in our study. The impure form of NS is more frequent in the adolescents than in younger patients and all adolescents with SRNS presented hypertension. Adolescents with impure NS at presentation, as well as a steroid-resistant status, have other lesions than minimal change disease. Gene mutations are often identified in SRNS with FSGS and this form of NS does not respond to immunosuppressive therapy and progress rapidly to ESRD. However, relapses are not expected following renal transplantation. The early genetic diagnosis in SRNS is time-consuming and expensive, but it is impor- 
tant for proper clinical management of the patients, prognosis and genetic counseling of the families.

\section{Acknowledgments}

We thank K. Tory, A. Fintha, M. Kardos, A. Szabo and Gy. Reusz for renal biopsy, histopathological exams and genetic testing.

\section{References}

1. Eddy AA, Symons JM. Nephrotic syndrome in childhood. Lancet. 2003;362(9384):629-639.

2. Valentini RP, Smoyer WE. Nephrotic syndrome, in Kher KK, Schnaper HW, Makker SP (eds): Clinical Pediatric Nephrology. $2^{\text {nd }}$ Edition. CRC Press, Informa UK Ltd. Abingdon, Oxon, 2007;155-194.

3. [ISKDC] International Study of Kidney Disease in Children. Nephrotic syndrome in children: prediction of histopathology from clinical and laboratory characteristics at time of diagnosis. A report of the International Study of Kidney Disease in Children. Kidney Int. 1978;13(2):159-165.

4. Gulati S., Sural S., Sharma RK., Gupta A, Gupta K. Spectrum of adolescent-onset nephrotic syndrome in Indian children. Pediatr Nephrol. 2001;16(12):1045-1048.

5. Gulati S, Sengupta D, Sharma RK et al. Steroid Resistant Nephrotic Syndrome: Role of Histopathology. Indian Pediatr. 2006;43(1):55-60.

6. Szabó JA, Kelen K, Reusz Gy. Nephrosis szindróma gyermekkorban. Gyermekgyógyászat. 2013;64(2):65-68.

7. [Guideline] International Study of Kidney Disease in Children [ISKDC] The primary nephrotic syndrome in children. Identification of patients with minimal change nephrotic syndrome from initial response to prednisone. A report of the International Study of Kidney Disease in Children. J Pediatr. 1981;98(4):561-564.

8. Mak SK, Short CD, Mallick NP. Long-term outcome of adult onset minimalchange nephropathy. Nephrol Dial Transplant. 1996; 11:2192-2201.

9. Tse KC, Lam MF, Yip PS, et al. Idiopathic minimal change nephrotic syndrome in older adults: steroid responsiveness and pattern of relapses. Nephrol Dial Transplant. 2003;18:1316-1320.
10. Chang JW, Tsai HL, Wang HH, Yang LY. Clinicopathological features and prognosis of Chinese children with idiopathic nephrotic syndrome between different age groups. Eur J Pediatr. 2009;168(10):1189-1194.

11. McKinney PA, Feltbower RG, Brocklebank JT, Fitzpatrick MM. Time trends and ethnic patterns of childhood nephrotic syndrome in Yorkshire, UK. Pediatr Nephrol 2001;16:1040-1044.

12. Mubarak M, Kazi Jl, Lanewala A, Hashmi S, Akhter F. Pathology of idiopathic nephrotic syndrome in children: are the adolescents different from young children? Nephrol Dial Transplant. 2012;27(2):722-726.

13. Borges FF, Shiraichi L, da Silva MP, Nishimoto El, Nogueira PC. Is focal segmental glomerulosclerosis increasing in patients with nephrotic syndrome? Pediatr Nephrol. 2007;22(9):1309-1313.

14. Banaszak, B and Banaszak, P. The increasing incidence of initial steroid resistance in childhood nephrotic syndrome. Pediatr Nephrol. 2012;27(6):927-932.

15. Popescu V, Olaru M. Sindromul nefrotic. in Popescu V. [ed] Algoritm de diagnostic si therapeutic in pediatrie. Vol. I, Ed. Medicala Almatea, Bucuresti. 2003;387-400.

16. Ponticelli C, Villa M, Cesana B et al. Can prolonged treatment improve the prognosis in adults with focal segmental glomerulosclerosis? Am J Kidney Dis, 1999;34:618-625.

17. National High Blood Pressure Education Program Working Group on High Blood Pressure in Children and Adolescents. The Fourth Report on the Diagnosis, Evaluation, and Treatment of High Blood Pressure in Children and Adolescents. Pediatrics. 2004;114(2):555 -576.

18. Pravitsitthikul N, Willis NS, Hodson EM, Craig JC. Non-corticosteroid immunosuppressive medications for steroidsensitive nephrotic syndrome in children. Cochrane Database Syst Rev. 2013;10:CD002290.

19. Esfahani ST, Madani A, Asgharian F. Clinical course and outcome of children with steroid-sensitive nephrotic syndrome. Pediatr Nephrol. 2011;26(7):1089-1093.

20. Mekahli D, Liutkus A, Ranchin B, et al. Long-term outcome of idiopathic steroid-resistant nephrotic syndrome: a multicenter study. Pediatr Nephrol. 2009;24(8):1525-1532.

21. Habashy D, Hodson EM, Craig JC. Interventions for steroid-resistant nephrotic syndrome: a systematic review. Pediatr Nephrol. 2003;18:906912. 